\title{
MUNCHENER HOCHSCHULSCHRIFTEN
}

\section{JOHANN SEBASTIAN BACH ERBE UND BESINNUNG}

\author{
von \\ RUDOLF VON FICKER
}

VERLAG VON R. OLDENBOURG

MUNCHEN 1950 



\title{
JOHANN SEBASTIAN BACH ERBE UND BESINNUNG
}

\author{
Rede \\ gehalten anläßlich des 478 . Stiftungstages \\ der Ludwig`Maximilians`Universität zu München (Ingolstadt) \\ am 1. Juli 1950 \\ von \\ Dr. phil. RUDOLF VON FICKER \\ Professor für Musikwissenschaft \\ an der Universität München \\ Begrüßungsansprache \\ des Rektors der Universität München \\ Professor Dr. WALTHER GERLACH
}

VERLAG VON R. OLDENBOURG MUNCHEN 1950 


\section{MUNCHENER HOCHSCHULSCHRIFTEN}

10 
BEGRÜSSUNGSANSPRACHE DES REKTORS DER UNIVERSITÄT

MÜNCHEN PROFESSOR DR. WALTHER GERLACH

Hochverehrte Gäste, Kommilitoninnen und Kommilitonen!

Im Namen des Senates der Ludwig-Maximilians-Universität heiße ich Sie alle herzlich willkommen.

478 Jahre sind wir alt - wir fühlen uns wesentlich jünger und begehen im Bewußtsein ewiger Jugend auch diesen Geburtstag.

Ein Geburtstag ist ein Fest, das die Familie vereinigt. So ist es uns eine große Freude, ein Mitglied des väterlichen Hauses Wittelsbach, dem wir Gründung und jahrhundertelange pflegliche Fürsorge verdanken, den Ehrenbürger unserer Universität, S. Kgl. Hoheit, Kronprinz Rupprecht hier zu begrüßen.

Auch die, welche ihre Mutterpflichten an uns üben, begrüße ich: Das Kultusministerium, vertreten durch Herrn Staatssekretär Dr. Sattler und Herrn Staatsrat Meinzolt, von dem wir materielle Nahrung erhalten und eine Besserung unserer Lebensbedingungen erhoffen, und die Stadt München, vertreten durch Herrn Bürgermeister Walther v. Miller, die uns seit I24 Jahren Dach und Schutz gewährt. Herrn Staatsrat Meinzolt, Herrn v. Miller und dem gesamten Stadtrat gilt auch ein dankbarer Gruß unserer Studierenden, ersterem für die Betreuung des Studentenwerkes, letzteren für ihre kulturelle Hilfe, insbesondere die Veranstaltung der Studentenkonzerte.

Ich danke herzlich dafür, daß der Oberbürgermeister von Ingolstadt, unserer Geburtsstadt, Herr Dr. Weber, und der Oberbürgermeister von Landshut, der Stadt, in der unsere Universität von I800-I825 blühte, Herr Lang, gekommen sind.

Ein besonderer Gruß gilt dem Vertreter der Bundesregierung, Herrn Bundesminister Schuberth, und dem stellvertretenden Ministerpräsidenten von Bayern, Herrn Staatsminister Dr. Josef Müller, ebenso den Vertretern der anderen bayerischen Ministerien, der staatlichen und städtischen Behörden.

Herzlich willkommen heißen wir die Herren Vertreter der Amerikanischen Regierung und die Herren Minister und Exzellenzen des britischen Königreiches, der Republiken Frankreich und Italien, des Königreiches der Niederlande und der Schweiz, die durch ihre Teilnahme ihr Interesse und ihre Hilfe bei unseren internationalen Austauschbestrebungen zeigen. 
Ich begrüße die Herren Vertreter der Kirchen, Herrn Präsidenten Dr. Rosenhaupt von der Bundesbahn mit einem Dank für seine stete Hilfsbereitschaft und Herrn Präsidenten Dr. Reimer vom Bundespatentamt.

$\mathrm{Da}$ wir Herrn Oberbürgermeister a. D. Scholl und seine Gattin unter uns haben, die Eltern unserer nicht vergessenen Kommilitonen, die mit ihren Freunden ihren Idealen für geistige Freiheit und Menschenwürde irdische Wünsche unterordneten, und Frau Professor Huber, die Witwe unseres Kollegen, der mit diesen Opfern ihrer Treue bis in den Tod verbunden blieb, - dieses ist uns eine ganz besondere Freude.

Ein letzter, aber nicht weniger herzlicher Gruß gilt unseren geistigen Brüdern und Schwestern: Herrn Präsidenten Mitteis von der bayr. Akad. d. Wiss., den Magnifizenzen Piloty von der Techn. Hochschule und Proesler von der Nürnberger Hochschule, den Herren Präsidenten Heger und Henselmann von den Hochschulen für Musik und für bildende Künste und dem Herrn Prorektor Professor Vogel von der Hochschule Dillingen, den Herrn Vertretern der Münchner Schulen und allen unseren Ehrenbürgerinnen und Ehrenbürgern.

Von den im letzten Jahre gestellten Preisaufgaben der theologischen Fakultät sind zwei Lösungen preisgekrönt. Die Fakultät hat der Arbeit mit dem Kennwort ,Videmus nunc per speculum in aenigmate: Tunc autem facie ad faciem. Nunc cognosco ex parte: Tunc autem cognoscam sicut et cognitus sum", den ersten Preis erteilt. Der Verfasser dieser Arbeit ist stud. theol. Karl Forster. Den zweiten Preis erhielt die Arbeit mit dem Kennwort „Ecce nova facio omnia“", Verfasser ist Viktor Käß. Namens der Universität beglückwünsche ich herzlichst die beiden Preisträger und hoffe, daß ihr Beispiel viele Kommilitonen anregen wird, sich im nächsten Jahre an den Preisaufgaben zu beteiligen.

Wir kehren in diesem Jahre zu dem früheren Brauch zurück, daß jede Fakultät eine Preisaufgabe stellt, welche bei dem Stiftungsfest erstmalig öffentlich bekanntgegeben wird. Die Preisaufgaben sind:

Theologische Fakultät: Volk und Haus Gottes in Augustins Lehre „Von der Kirche".

Juristische Fakultät: ,Welchen Einfluß auf die Frage der Zulässigkeit oder der Unzulässigkeit des ordentlichen Rechtswegs hat der $\oint 22$ der in der amerikanischen Zone erlassenen Gesetze über die Verwaltungsgerichtsbarkeit und der Verordñung Nr. ${ }^{6} 5$ der britischen Militärregierung über die Verwaltungsgerichtsbarkeit?"“ 
Staatswirtschaftliche Fakultät : „Die betriebswirtschaftliche Problematik bei der Entflechtung der industriellen Großunternehmungen."

Medizinische Fakultät : „Die Cytochemie der Leukämie.“

Tierärztliche Fakultät : „Durch Versuche an verschiedenen Tierarten soll geprüft werden, ob sich Curare in der Tiermedizin zu therapeutischen Zwecken eignet."

Philosophische Fakultät : „Die lateinischen cognomina in ihrer Bedeutung für das Vulgärlatein und die romanische Sprachentwicklung ". Näheres kann im Philosophischen Dekanat, bzw. im Rektorat erfragt werden.

Naturwissenschaftliche Fakultät : „Die Darstellung eines Lithium-Magnesium-Hydrids."

Meine Damen und Herren!

Am 26. Juni 1472 wurde unsere von Herzog Ludwig dem Reichen, Pfalzgraf bei Rhein und Herzog von Nieder- und Oberbayern begründete Universität durch den Landshuter Rat Martin Mair feierlich eröffnet.

Die geistige Lage dieser Zeit war in manchem der heutigen vergleichbar - man hatte wenig Glauben an eine freundliche Zukunft, man befürchtete einen die Welt vernichtenden Brand. Die geistigen und politischen Erschütterungen der Reformation warfen ihre Schatten voraus, die Kämpfe zwischen kirchlicher und weltlicher Macht griffen auf das geistige wie das tägliche Leben über, die soziale Ordnung schwankte. „Unsere Nation, zerrissen und zerschlagen, findet zu keiner Stunde Ruh", hatte Gregor von Heimburg, der Syndikus des Rates der Stadt Nürnberg, gerufen - er starb, als unsere Universität gegründet wurde.

Dem gegenüber sollte die Stfitung der ,,hohen gemein würdigen und freien" Universität der Ausdruck einer optimistischen Bejahung des Fortschrittes sein:

„Daß man nun fürbaß ewiglich daselbst lesen, lehren und lernen soll alle göttlich erlaubte und gewöhnliche Kunst von natürlichem Wesen und guten Sitten, von geistlichen und weltlichen Rechten, von der Arznei und den freien Künsten."

Die Universität hatte also von vornherein die vier Grundfakultäten. Durch sie sollte

„das Recht und gemeiner Nutzen gepflanzt, auch die so von niederer Geburt herkommen, zu hohen Würden und Stand gefördert werden". 
Mit dieser sozialen Forderung greift Ludwig der Reiche weit in die $\mathrm{Zu}$ kunft, und wir glauben, daß wir heute die von unserer Zeit geforderten Wege im Sinne unseres Stifters erfolgreich suchen.

Und auch darin folgen wir seinem Willen: Der gemeine Nutzen, den unser Stifter hier von uns gepflanzt haben will, soll nicht nur unserem Lande, unserer Nation dienen. Ausdrücklich wirdin der Gründungsurkunde der europäische Charakter der neuen Universität bezeugt; namentlich werden alle Stämme und Länder unseres Erdteils genannt, aus welchen Studenten und Professoren erwartet werden; und in der Tat finden wir bald unter ihnen Männer hoher Bedeutung aus vielen fremden Ländern. Auch hier hoffen wir auf eine weitere glückliche, weltoffene Entwicklung unserer alma mater.

Aber noch mehr: Die Zuversicht unseres Stifters läßt auch uns über Trümmer und Trübsal die Blicke auf neue Ziele richten, gegründet auf die unvergänglichen Werte unserer Geistesgeschichte. Das möge heute darin zum Ausdruck kommen, daß wir die Feier unseres Stiftungstages mit dem Gedenken an Johann Sebastian Bach, einen der Titanen im Reiche der Kunst, verbinden.

„,Denn nur die Kunst und die Wissenschaft erhöhen den Menschen bis zur Gottheit." Mit diesem Worte Beethovens treten wir in das 479. Jahr unserer Universität. 


\title{
JOHANN SEBASTIAN BACH ERBE UND BESINNUNG
}

\author{
Festrede \\ von \\ RUDOLF VON FICKER
}

Gehalten beim 478. Stiftungsfest

der Ludwig/Maximilians`Universität München (Ingolstadt) am 1. Juli 1950 
Es mag vielleicht unangebracht erscheinen, in dieser Stunde, die dem Gedächtnis Joh. Seb. Bachs gewidmet sein soll, auch jenes großen Zeitgenossen zu gedenken, den die Mit- und unmittelbare Nachwelt hoch über den Meister stellte: Georg Friedrich Händel. Dies soll nicht deshalb geschehen, um die bisweilen noch heute diskutierte, müßige Streitfrage zu berühren, welchem der beiden größten Musiker des späten Barock der Vorrang gebühre: Es soll vielmehr nur versucht werden, durch einen Seitenblick auf die Erscheinung Händels die Erkenntnis der Eigenart von Bachs Kunst zu erleichtern und vielleicht auch klarer zu gestalten. Denn die Laune des Zufalles hatte es gefügt, daß eine seltene Übereinstimmung bestimmender Faktoren, wie Herkunft, Begabung und Erziehung eine völlig gleichartige Entwicklung der jugendlichen Meister zu verbürgen schien: Beide sind in demselben Jahre 1685 geboren. Sie entstammen demselben mitteldeutschen Ursprungslande der lutherischen Konfession, aus dem seit dem I6. Jahrhundert jene unvergleichlichen Generationen von Organisten hervorgegangen waren, deren lange Reihe dann Bach und Händel als die letzten und gewaltigsten Beherrscher des königlichen Instrumentes abschließen sollten. Kaum dem Knabenalter entwachsen widmeten sie ihre frühgereiften Kräfte dem anspruchsvollen kultischen Dienste ihres Glaubens. Daneben empfingen sie von der nach französischem und italienischem Vorbild eingerichteten Musikpflege der kleinen sächsisch-thüringischen Fürstenhöfe wertvolle Anregungen für ihr künftiges Schaffen auf dem Gebiete der weltlichen Instrumentalformen.

Vom I8. Lebensjahr ab nimmt jedoch die Entwicklung Händels plötzlich einen anderen, völlig unerwarteten Verlauf. Seine impulsive Natur drängt mit aller Macht hinweg aus der in kultischer Enge erstarrten Kunstsphäre der Heimat. Hingerissen von der fantastischen Erscheinung der Barockoper, die er in Weißenfels und Hamburg kennenlernt, vollzieht er in kühnem Entschlusse die Wandlung vom Kirchen- zum Opernkomponisten. Er verläßt endgültig die Heimat und nimmt vorerst in Italien den Wettbewerb mit den bedeutendsten einheimischen Meistern 
auf deren ureigenem Kunstgebiete erfolgreich auf. Als letzter und größter Vertreter der italienischen Barockoper verhilft er dieser dann auch in England, das ihm fortan zur dauernden Heimat wird, zum Siege.

Nach Jahren triumphaler Erfolge vermag jedoch selbst das Genie Händels nicht die wachsende Opposition jener englischen Kreise zu beschwichtigen, deren nüchterner, rationaler Sinn sich gegen die unnatürliche Scheinwelt der alten Barockoper richtete, mit ihren stereotypen Requisiten unglaubhafter Zauberei und intrigenvoller Verwicklungen, der Übersteigerung aller triebhaften Leidenschaften bis zur sinnlosen Unvernunft. Nach empfindlichen Rückschlägen vollzieht Händels wendig-beweglicher Geist die entscheidende letzte Wandlung, indem er jene musikalische Kunstgattung neu gestaltet, die dem puritanisch geläuterten Empfinden der angelsächsischen Mitwelt eine überwältigende künstlerische Formung schuf: es entstehen in den beiden letzten Jahrzehnten seines Schaffens die großen Oratorien über biblische Texte.

Die Annahme, Händel hätte hier wieder zum religiösen Ursprung seiner Kunst zurückgefunden, trifft allerdings nur in einem sehr bedingten Grade zu. Denn es handelt sich bei seinen Oratorien um Äußerungen einer durchaus modernen religiösen Gefühlshaltung, die ausschließlich menschlichem Gewissenszwange entsprungen, nun jeder kirchlich-konfessionellen, geschweige denn liturgischen Bindung völlig entzogen ist. Es ging hier Händel darum, der Gemeinschaft der Zuhörer in den Schicksalen des auserwählten Volkes Israel sowohl das ethische Vorbild, sowie auch ein Spiegelbild des eigenen moralischen Gewissens vorzuhalten und hierdurch jene seelische Identifizierung des teilnehmenden Subjektes mit dem künstlerischen Darstellungsobjekt zu erzwingen, die dann das im eigentlichen Sinne Bedeutsame und Neue der musikalischen Entwicklung seit der Klassik ist.

Die spontane, anhaltende Wirkung des Händelschen Oratoriums beruhte daher nicht allein auf den hohen musikalischen Qualitäten dieser Werke, die ja auch in seinen Opern vorhanden sind, sondern darin, daß hier trotz der biblischen Stoffe die Humanitätsanschauungen der neuen Vernunftlehre des Rationalismus auf musikalischem Gebiete zum erstenmal eine vollgültige künstlerische Prägung gefunden hatten. Auf der unerschütterlichen Grundlage dieser Werke, zumal des ,, Messias “ Händels, sind dann alle großen Chorschöpfungen seit der Klassik erwachsen, sowohl Mozarts „Requiem“, Haydns ,,Schöpfung“", wie auch die „,Missa solemnis" Beethovens, der selbst noch in seinen späten Schaffensjahren Händel als den größten aller Meister bezeichnete. Als Händel um die Zeit, da Bach das Zeitliche segnete, erblindete und der schöpferischen Tätigkeit entsagen mußte, konnte er bis zu seinem Todesjahr I759 wenigstens noch die wahrhaft königlichen Ehrungen entgegennehmen, die ihm besonders 
das englische Volk bereitete und dann über den Tod hinaus unverändert bewahrte.

Die Schicksale und künstlerischen Ziele J. S. Bachs sind jenen Händels völlig entgegengesetzt. Sein Leben und Wirken spielte sich ohne bedeutsame Ereignisse im engen Umkreis der Heimat ab, kaum beachtet von der übrigen Welt. Er weicht von den ihm seit der Jugend vorgezeichneten Bahnen bis zum Ende nicht ab, hält an den überlieferten handwerklichen Techniken unentwegt fest und ist bestrebt, diesen in einer letzten Steigerung noch das Äußerste abzugewinnen, obwohl sich seine Kunstgenossen schon längst viel einfacherer und vor allem gefälligerer Praktiken bedienten. Anläßlich seiner Tätigkeit am Hofe von Cöthen hätte sich ihm zwar die Gelegenheit eröffnet, dem anstrengenden und undankbaren Frondienst eines Kantors zu entgehen und - ähnlich wie Händel — die kirchliche mit der weltlichen Kunstsphäre zu vertauschen. Daß er hierfür die Eignung hatte, beweisen seine bekannten Instrumentalwerke im neuen italienischen und französischen Geschmack, die fast alle in dieser Frühzeit entstanden sind, so u. a. die Brandenburgischen Konzerte, die vier Orchester-Suiten, von welchen Sie heute jenes Werk hören ${ }^{1}$ ), in welchem die französische Art wohl die vollendetste Ausprägung gefunden hat. Obwohl die idyllischen Cöthener Jahre die glücklichsten seines Lebens waren, ordnet sich dann Bach mit der Übernahme des Leipziger Thomaskantorates im Jahre I723 doch wieder der ererbten Bestimmung der Vorfahren seines Geschlechtes ein, auch in der Kunstübung nichts anderes sein zu wollen als der treue Diener seines Glaubens.

Es ist bekannt, wie wenig Anerkennung Bach in Leipzig von seiten seiner, wie er einmal schreibt ,wunderlichen und der Music wenig ergebenen Obrigkeit" empfängt, mit welcher Verbissenheit, aber völlig vergeblich er um die Verbesserung der musikalischen Verhältnisse kämpft, die ihm nie gestatteten, seine monumentalen Kantaten und Passionen in einer künstlerisch halbwegs entsprechenden Besetzung aufzuführen. Unter den Mitbürgern ist es nur ein kleiner Kreis, der die Größe dieser nach altmodisch-überlebten Formen gestalteten Werke des Meisters zu ahnen vermochte. Und als Bach nach Jahren zunehmender Verbitterung und Vereinsamung I750 starb, blieb bei den Nachfahren und sogar bei den eigenen Söhnen, die viel mehr Ruhm ernteten wie der Vater, nur mehr die Erinnerung an ihn lebendig als eines gelehrten Fugisten, dessen Werke schon zu seinen Lebzeiten unmodern geworden waren, der Nachwelt vollends nichts mehr zu sagen hatten.

So schien auch seinem Erbe das Schicksal bestimmt, der Vergessenheit anheimzufallen, das vor ihm allen großen Meistern der Musik beschieden

1) Suite in h-moll, gespielt vom Orchester der Bayer. Staatsoper unter Leitung von G. Solti. 
war, wann immer eine Wandlung des Geschmackes das Interesse am Hergebrachten erlahmen ließ, die traditionellen Bahnen der Interpretation unsicher wurden, sich allmählich verloren und damit die lebendige Beziehung zum Kunstwerk erlosch.

Zwar empfanden in der Folge einige Auserwählte, unter diesen ein Mozart und Beethoven, noch immer die geheimen Kräfte, die sich ihnen in den wenigen damals fast allein bekannten Fugen- und Motettenwerken Bachs eröffneten und die bekanntlich nicht ohne Einfluß auf das späte Schaffen dieser Klassiker blieben. Aber es erschien auch diesen Meistern nötig, die erborgten Formen mit einem neuen geistigen oder - wie Beethoven einmal sagt - ,poetischen" Element zu erfüllen und hierdurch völlig abzuwandeln. Die besonderen Kräfte geistiger Art, welchen die Kunst des Thomaskantors noch verhaftet war, waren auch der Klassik nicht mehr faßbar.

Eine Wendung brachte erst die Romantik, deren künstlerische Ideale dem Wesen der Tonsprache Bachs eigentlich ganz ferne zu liegen schienen. Aber die schwärmerische Begeisterung der Romantiker für die Vergangenheit, die ihnen eine Welt unberührter Vollkommenheit dünkte, hatte die Entstehung eines vorerst mehr idealistischen, als sachlich-kritischen Historismus begünstigt, der sich auch auf dem Gebiete der Musik auswirkte und hier durch den neu erwachten religiösen Pietismus der Zeit eine nachdrückliche Stärkung empfing. Im katholischen Lager war es besonders Palestrina, an dessen a capella-Kunst man den Idealtypus einer „reinen“" geistlichen Tonkunst zu demonstrieren versuchte. Im protestantischen wandte sich hingegen die Aufmerksamkeit in stets steigendem Grade der zeitlich näher liegenden Erscheinung Bachs zu. Seit der denkwürdigen ersten Wiederaufführung der Matthäus-Passion durch Mendelssohn im Jahre I829, seit dem Bekanntwerden der großen Kirchenkantaten wurde eine bis dahin fast unbekannte Seite von Bachs Kunst offenbar, die das Empfinden der Zeit ungleich stärker berührte, als die wenigen, bisher fast allein zugänglichen polyphonen Instrumentalwerke des Meisters. Es war besonders die naiv-volkstümliche, wenn auch bisweilen als drastisch und bizarr empfundene Sprache der barocken Texte dieser mächtigen geistlichen Chorwerke, die gemütsinnige Einfachheit der Choräle einerseits, die dramatische Vehemenz der Rezitative und der Chöre anderseits, in welchen Eigenschaften die Romantik Züge ihrer eigenen künstlerischen Sehnsucht erblicken zu können vermeinte.

Aber erst in der zweiten Hälfte des I9. Jahrhunderts wurde das Werk des Meisters in seiner umfassenden Größe und Eigenart völlig erschlossen. Von $185 \mathrm{I}$ - I900 erstand die große Gesamtausgabe seiner erhaltengebliebenen Kompositionen, I873/80 erschien die klassische Biographie des Meisters von Philipp Spitta als erste große Leistung der modernen 
musikgeschichtlichen Forschung. Aber die Beschäftigung mit Bachs Kunst blieb nicht eine antiquarische Angelegenheit, wie dies sonst in der Musik bei Neuausgrabungen der Werke vergessener Meister gewöhnlich der Fall ist. Viel bedeutsamer wurde die seitdem stets wachsende Pflege seiner Werke, die der Erscheinung Bachs eine Aktualität verschaffte, die jener Händels und der großen Meister seit der Klassik kaum nachstand, ja diese bisweilen noch zu übertreffen vermochte.

Es erscheint unmöglich, zur Erklärung dieser seltsamen Tatsache Gründe rein musikalischer Art allein ins Treffen zu führen, wie dies die evolutionistische Musikanschauung des vergangenen Jahrhunderts versuchte, die in Bach den "Urvater der Harmonie", den ersten ragenden Markstein der musikalischen Entwicklung oder gar den Wegbereiter der nachfolgenden Klassik und Romantik erblickte. Träfe diese Auffassung zu, dann hätte es unmöglich geschehen können, daß sein Schaffen gerade bei den Nachfolgern fast unbeachtet und unverstanden blieb und erst im Ausklang der klassisch-romantischen Richtung zu dauernden Ehren gelangte. Und es wäre schwer zu verstehen, warum die Geltung von Bachs Werk selbst noch in unserer Zeit eine so unantastbare geblieben ist, obwohl sich heute in fast allen grundsätzlichen Belangen des Musikalischen eine völlige Umwertung vollzogen hat, die den materialen Gegebenheiten seiner Tonsprache völlig zu widersprechen scheint.

Solche Überlegungen vermögen eher die Annahme zu rechtfertigen, daß mit dem Erbe Bachs bestimmte geistige Kräfte überliefert sind, die der Musik unserer Zeit abhandengekommen sind, deren sie indessen als Ergänzung oder sogar als Ersatz dringend bedürfte. Lassen sich aber solche besondere geistige Kräfte im Schaffen des Meisters feststellen? Von welcher Art sind sie und woher stammen sie?

Albert Schweitzer hat in seinem bekannten Werke über Bach vornehmlich die ",tonmalenden" Elemente im Schaffen des Meisters herausgearbeitet, die bereits die Romantik vorahnend empfand. Aber in der Einleitung des Buches spricht er die bedeutungsvollen Worte aus: ,So ist Bach ein Ende. Es geht nichts von ihm aus, alles führt nur auf ihn hin." Obwohl diese Feststellung nur in dem Sinne gemeint ist, daß Bach in der Entwicklung der Musik des deutschen Protestantismus das unüberbietbare Endglied darstellt, so kommt ihr doch eine viel umfassendere Bedeutung zu.

Denn wir erblicken heute in Bach auf dem Gebiete der Musik den letzten Repräsentanten einer geistigen Welt, deren Grundlagen schon seit der Wende von der Renaissance zum Barock allmählich preisgegeben worden waren, die hingegen in der engen Enklave des mittel- und nord- 
deutschen Protestantismus bewahrt blieben und hier in ihrer Verbindung mit der allgemeinen Formensprache der Zeit jenen Sondertypus eines deutschen Barocks erstehen ließen, der - in genauer zeitlicher Übereinstimmung mit der Baukunst eines Balthasar Neumann - in Joh. Seb. Bach seinen ragenden Höhepunkt gefunden hat. Denn bei den deutschen Kantoren des Barocks war das künstlerische Schaffen noch durchweg im Sakralen verankert geblieben. Als dienendes Organ einer höheren, übersinnlichen Macht mußte der Künstler seine Tätigkeit als transzendentalen Auftrag in einem sozusagen handwerklichen Sinne auffassen, der ihm zugleich die Möglichkeit bot, bei der Verwirklichung der ihm gestellten Aufgabe alle Kräfte individueller Fantasie und Kunstfertigkeit einzusetzen. Es ist also in der Musik des deutschen Barocks noch dieselbe innere Haltung bewahrt, die bereits das künstlerische Schaffen des hohen Mittelalters kennzeichnete. Denn schon in der Renaissance des I6. Jahrhunderts werden sonst überall die transzendenten Bindungen gelockert, setzt jetzt die Ära eines selbstherrlichen Künstlertumes ein, das in sich selbst die Gloriole des Göttlichen zu bergen vermeint.

Solche Zusammenhänge geistiger Art im Werke Bachs wurden bisweilen mehr intuitiv geahnt, als klar gesehen. So hat Richard Benz einmal gemeint, daß sich die Gotik in der Musik um einige Jahrhunderte verspätet habe und erst mit dem Thomaskantor in Erscheinung getreten sei. Er spricht daher stets von der gotischen Tonbaukunst Bachs. Eine solche Kennzeichnung der Kunst Bachs ist allerdings, wie wir heute wissen; historisch nicht haltbar. Aber sie enthält insofern etwas Richtiges, als in der Tat die Musik des deutschen Barocks in ihrer geistigen Haltung noch bestimmende Züge aufweist, die sich kaum von jener der Gotik unterscheiden.

Denn hier wie dort bestand die zentrale Aufgabe des Künstlers in der musikalischen Ausgestaltung des kirchlichen Kultus, jedoch nicht in Gestalt jener subjektiven religiösen Bekenntnismusik, die sich überall sonst seit der Renaissance entwickelt hatte, sondern einer Musik, die noch von einer gleichsam dogmatischen Strenge war, indem hier die Wahrung der überlieferten liturgischen Gehalte als der unwandelbaren Glaubens-Symbole für unbedingt verpflichtend erachtet wurde. Dieses Symbol war in der Gotik der uralte gregorianische Choral, im deutschen Barock hingegen der protestantische Choral, das von Luther begründete und der ganzen Gemeinde vertraute evangelische Kirchenlied, das einen wesentlichen Bestandteil der gottesdienstlichen Ordnung bildete. Schon Luther erkannte nicht nur die hohe ethische, sondern auch propagandistische Kraft dieses kirchlichen Gemeinschaftsliedes. Und der Jesuit Conzenius klagte, Luthers Gesänge hätten mehr Seelen umgebracht, als alle seine Schriften und Reden. 
Wie bei den Kantoren vor und neben ihm, bildet der Choral auch noch für Bach die unerschütterliche Grundlage und den Ausgangspunkt des künstlerischen Schaffens, und zwar nicht nur in den ideellen, sondern auch in den technisch-musikalischen Belangen. Denn dieses fremde, unscheinbare Choralsymbol ist gleichsam der atomhafte musikalische Kern, an dem sich nun in einer gewaltigen Reaktion die unbändige Kraft der Fantasie und Kunstfertigkeit des Meisters entzündet und entfaltet, deren Ergebnis die riesenhafte Welt der Orgelchoräle, Kirchenkantaten und Passionen darstellt. Stets bleibt hier der Choral die geistige Mitte, sei es nun, daß er in schlichter Formung gleich festen Pfeilern den vielteiligen Kunstbau zusammenhält, der erdgebundenen Erregtheit der Rezitative, Arien und polyphonen Chorsätze die transzendente Unsinnlichkeit des tongewordenen göttlichen Symboles als ordnendes und zugleich bändigendes Element beigesellt, oder in kunstvoller Umbildung den Formablauf wie ein kaum sicht- und hörbares geistiges Band durchzieht. Alle die vielfältigen kunstmäßigen Möglichkeiten der harmonischen, melodischkontrapunktischen und rhythmischen Ausformung, die Bachs polyphonen Stil im allgemeinen bedingen, wurden von dem Meister vor allem in der choralgebundenen Orgelkunst entwickelt, die sein Schaffen von der frühesten Jugend bis zum Lebensende begleitete, deren kunstmäßige und technische Errungenschaften er dann auf alle übrigen Formgattungen geistlicher und weltlicher Art überträgt.

Es ist also noch bei Bach völlig unbewußt dasselbe künstlerische Verfahren bewahrt, das schon den Musiker der frühen Gotik veranlaßte, aus der symbolgeladenen Kernformel des alten gregorianischen Chorales die mächtigsten musikalischen Gebilde zu entwickeln, und zwar auch hier unter Verwendung aller sinnlich-naturalistischen Gestaltungsmittel, über welche die Zeit verfügte. Denn damals wurden die Begriffe "Geistlich“ und „Weltlich" noch nicht als Gegensätze empfunden. In der Motette des I3. Jahrhunderts erklingen häufig profane Texte von bisweilen frivolster Prägung gleichzeitig zum spirituellen Symbol eines lateinischen Choralspruches. Und aus derselben Empfindungsart gesellt noch ein halbes Jahrtausend später die Bachsche Kirchenkantate dem religiösbesinnlichen Choral unbedenklich das kreatürlich-schwülstige Pathos spätbarocker Dichtungsart und läßt dazu in der Musik alle Grade subjektiver Stimmung von melancholischer Trauer bis zu ausgelassenster Fröhlichkeit aufklingen.

Schon der Pietismus der Zeit Bachs verdammte ein solches Verfahren als völlig unkirchlich, das dann auch die Ursache war, daß nach dem Tode des Meisters sein Kantatenwerk der Vergessenheit anheimfiel und auch heute den alten Platz nicht mehr zurückzugewinnen vermochte. Denn die aufgeklärte Menschheit des I8. und I9. Jahrhunderts konnte nicht 
mehr verstehen, daß hier bei Bach - wie schon in der Motette, aber auch in der Kathedralkunst der Gotik! — noch eine Glaubenshaltung lebendig war, die in der Vorstellung jener Majestas Ecclesiae begründet war, die sich durchaus die Kraft zutraute, ebenso die Niedrig- und Nichtigkeiten des profanen Alltages, wie auch allen schreckhaften Spuk dämonischer Gewalten an sich zu ziehen, um sie mit der Allgewalt göttlicher Symbole zu durchsetzen und in dieser abgewandelten, verklärten Gestalt dem natürlichen Leben zurückzugeben.

Aber diese Zusammenhänge mit einer fernen Vergangenheit bestehen sogar in Übereinstimmungen äußerlicher Art. Denn wenn Bach, gleich den übrigen Kantoren der Epoche, ganze Zyklen umfangreicher Kantaten für die Sonntage des jeweiligen Kirchenjahres komponierte, so entspricht dies z. B. genau dem Verfahren der großen Meister von Notre Dame um I200, die ähnliche Zyklen geschaffen haben, nur mit dem einzigen Unterschied, daß hier noch der alte gregorianische Choral als Grundlage diente. Erst seit der Renaissance wird in der kirchlichen Kunstmusik der Zusammenhang mit dem Choral, dessen künstlerische Eigenschaften der Zeit bereits fast völlig verlorengegangen waren, allmählich preisgegeben, während dann die alte kunstmäßige Tradition auf der Basis des neuen deutschen Chorales von den Kantoren des Barocks weiter- und mit Bach zu Ende geführt wird.

Was daher dem hohen Mittelalter und dann auch noch dem deutschen Barock die entscheidende Signatur verleiht, ist der den Nachfahren unverständliche Dualismus zwischen der stets gegenwärtigen Idee des Göttlichen, symbolisiert durch den entlehnten Choral, und dessen naturalistischer Umbüllung oder Umgestaltung, wobei der Anwendung aller subjektiven künstlerischen Freiheiten keine Grenzen gesetzt sind.

Dieser Dualismus bedingt jedoch auch das Musikalisch-Technische dieser Kunst. Denn der Widerstreit und gleichzeitig die harmonische Ausgleichung verschiedener, scheinbar selbständiger Stimmen hat nirgends in der Musik eine so konsequente und vollendete Gestaltung gefunden, wie in der Polyphonie Bachs. Auch sie ist nicht eine bloße musikalische Stilerscheinung oder ein Akt künstlerischer Eigenwilligkeit. Denn in ihren tieferen Ursachen ist auch sie im Zerspalteten der menschlichen Natur des protestantischen Barocks begründet, die noch mit aller Stärke die Abhängigkeit des Irdischen vom Überirdischen, des Sinnlichen vom Übersinnlichen empfand, ohne im geringsten eine Annäherung oder Ausgleichung der gegensätzlichen Kräfte $\mathrm{zu}$ wagen oder überhaupt als wünschenswertes künstlerisches und geistiges $\mathrm{Ziel} z u$ betrachten.

Es ist aber begreiflich, daß die Kunst Bachs, anders wie jene Händels, als unmodern und reaktionär fast völlig unbeachtet blieb. Denn die neue 
Vernunftlehre des Rationalismus leugnete jeden Einfluß des Religiösen auf den Menschen und mußte daher auch für das künstlerische Schaffen die Wirksamkeit transzendenter Bindungen verneinen. Auch den Musikästheten galt als einzige Parole nur mehr die "Nachahmung der Natur", worunter sowohl die naive Darstellung äußerer Naturvorgänge, als auch jene der menschlichen Affekte und Leidenschaften verstanden wurde. Auch Bach blieb von diesen neuen Formulierungen nicht unberührt, soweit sie nicht die für ihn unerschütterliche Zone des Religiösen betrafen.

So hatte die Musikästhetik der Zeit ein System ausgeklügelt, in welchem die Stilisierung der Affekte in der Musik durch genau fixierte rhythmische Bewegungsmotive, entsprechende Wahl der Tonarten u. dgl. festgelegt war, so daß bereits das optische Notenbild den jeweiligen Affektgehalt eines Tonstückes erkennbar machte. Wir wissen insbesondere seit dem Bach-Werke Schweitzers, welch reichen Gebrauch der Meister von dieser zeichnerischen Technik machte. So - um nur ein Beispiel zu nennen wenn er in einer Kantate den drastischen Vorgang der Textphrase: „,so wie ein Teufel den andern frißt" durch die engführende Verschlingung eines abschließenden Motives gleichsam als tönende Zeichnung illustriert.

Diese nachahmend-affekthafte Seite der neuen Lehre fand anderseits in der Forderung einer von moralischen Gesetzen diktierten vernunftgemäßen Ordnung ihre Ergänzung und zugleich Zügelung. In der deutschen Ästhetik des Hochbarock führten dann diese Bestrebungen sogar zu einer Verbindung von Musik und Mathematik, so daß Lorenz Christoph Mizler, der Begründer der Leipziger ,,Sozietät musikalischer Wissenschaften“, der auch Bach angehörte, Musik als eine ,unbewußte Rechenübung der Seele" definieren konnte. ${ }^{1}$ ) Wer sich einmal in den planmäßig durchdachten Konstruktivismus des ,Wohltemperierten Klaviers" oder gar der ,,Kunst der Fuge " versenkt hat, dem dürfte kaum entgangen sein, wie tief und eigenartig Bach auf seine Weise solchen Forderungen der ,,ratio" im künstlerischen Schaffen zu genügen vermochte.

Schien so Bach solchen ästhetischen Forderungen der Zeit immerhin Rechnung getragen zu haben, so mußte er doch die Auswirkung der grundsätzlichen Ideen des Rationalismus als völlig unvereinbar mit seiner eigenen Kunstart erachten. Denn besonders die französischen Vertreter der Lehre erblickten in der Musik nichts anderes als eine ,natürliche Sprache" oder, wie es dann Roussean formulierte, ,,ein aus den Sonorelementen einer Ursprache herausgeholtes Etwas". Und so wurde jetzt die einfache, affektmäßig gestaltete Melodie, höchstens gestützt durch einfache Akkorde, das Ideal der Epoche, das bereits in der italienischen Arie besonders sinnfällig erfüllt war und bekanntlich selbst noch den

1) W. Serauky ,Die musikalische Nachahmungsästhetik im Zeitraum von I700-1850“. 
Ansprüchen der nahen Klassik eines Haydn und Mozart am vollkommensten entsprach. Auch Händel hatte schon seit langem den neuen Erfordernissen einer einfach-natürlichen. Tonsprache immer stärker Rechnung getragen und hat hierdurch auch von der musikalisch-kunstmäßigen Seite her die breite Auswirkung besonders seiner Chor-Oratorien erleichtert.

Nur Bach hielt als einziger und letzter Vertreter des Spätbarocks unverrückbar an der veralteten polyphonen Technik fest. Er überträgt sie sogar auf jene von ihm übernommenen Formen, welche unter der Einwirkung der neuen ,,natürlichen“ Musikanschauung in Italien entstanden waren, wie besonders die Arie und das Konzert, die bei Bach infolge der kontrapunktisch-polyphonen Umhüllung einen gänzlich veränderten Sinn empfangen. Denn ein melodischer Gedanke kann für Bach nie allein bestehen, sondern nur in der Gefolgschaft und im harmonischen Zusammenwirken mit gleichberechtigten anderen Gedanken. Gerade aber dieses polyphone Verfahren Bachs, das uns heute als unvergleichliche künstlerische Bereicherung erscheint, mußte dem Empfinden seiner Zeit, das nur den einfach-natürlichen Affekt gelten ließ, als sinnlose, gekünstelte Hypertrophie gelten, als eine ,invention gothique“, wie Rousseau die Polyphonie bezeichnete.

Es war daher nicht ein Mißverstehen seiner Kunst oder gar die Verständnislosigkeit der Mit- und Nachwelt, welche Bachs Werk der Nichtbeachtung und schließlich der langen Vergessenheit anheimfallen ließ. Die Ursache hierfür lag an Bach selbst, an seinem starren Festhalten an geistigen und künstlerischen Ideen, die der Zeit bereits völlig fremd geworden waren, weil sie die transzendente Gebundenheit seiner Erscheinung und den Dualismus seiner Kunstauffassung nicht mehr zu begreifen vermochte.

Denn schon mit Händel tritt jener neue Typus des modernen Musikers in Erscheinung, dessen Bestimmung jetzt darin beschlossen ist, die schöpferischen Kräfte in freier innerlicher Auseinandersetzung mit der bannenden Gewalt genialer Eingebung zu bezwingen. Es ist dies eine Entwicklung, die in Italien bereits in der Renaissance eingesetzt hatte. Aber sie gelangt in der Musik erst jetzt zur vollen Auswirkung, nachdem sie nun endgültig den kirchlichen oder höfischen Rahmen verlassen hatte, in welchem sie bisher einer einschränkenden Kontrolle unterstand. Von nun ab erfährt die musikalische Wiedergabe eine Vorlagerung an eine neutrale, sozusagen ,,museale" Stätte, den modernen Konzertsaal, dessen Bestimmung dem dargebotenen Kunstwerk keine zweckhaften, konventionellen Bindungen auferlegt. 
Diese erst in der Klassik zum vollen Durchbruch gelangte Zentrierung der gestaltenden Kräfte im Subjektiv-Menschlichen, die Freiheit, das Kunstwerk, ohne Bezugnahme auf etwas Außen- und Höherstehendes, aus der drängenden Fülle innerlicher Nötigung allein erstehen zu lassen, dieser dem I9. Jahrhundert so selbstverständliche und unentbehrliche Subjektivismus im Kunstschaffen hat besonders in der Musik, als der unmateriellsten der Künste, jene absolute Kunsthöhe bewirkt, die seit der Klassik zu Einzelleistungen von unüberbietbarer Größe geführt hat.

Wenn auch hier die Humanitätsideale der Aufklärung noch überall nachwirkten, ja jetzt erst ihre volle künstlerische Erfüllung fanden, so wurden diese doch von jedem der großen Meister des Jahrhunderts stets in anderer Sicht gesehen und künstlerisch anders gestaltet. Diese Freiheit des schöpferischen Genies begünstigte und ermöglichte erst jene ungeheuere Evolution, die sich jetzt sowohl hinsichtlich der Steigerung der subjektiv-emotionalen, wie auch der technisch-musikalischen Elemente innerhalb der knappen Spanne eines Jahrhunderts vollzieht. Es war ferner der trügerische Glaube an den ewigen Fortschritt der Kunst, der - besonders in der zweiten Jahrhunderthälfte - die Entwicklung unablässig weitertreibt. Alle musikalischen Mittel und Gehalte erfahren nun eine minutiöse Differenzierung und Steigerung, bis jenes unvermeidliche Endstadium erreicht ist, in welchem alle Kräfte, die früher einer tausendjährigen Entwicklung abendländischer Musik genügt und standgehalten hatten, der allmählichen Auflösung entgegenzugehen oder in ihr Gegenteil verkehrt zu sein scheinen.

Es ist eine der seltsamsten Erscheinungen, daß inmitten dieser Loslösung von den herkömmlichen materialen und künstlerischen Werten gerade die Gestalt des alten Thomaskantors eine Geltung erlangte, wie nie zuvor, daß sein Werk an Gegenwartsbedeutung das aller anderen großen Meister seit der Klassik und Romantik beinahe zu überstrahlen vermochte. Diese Tatsache ist nicht aus musikalisch-sachlichen Gründen allein zu erklären, wie mitunter angenommen wird. Wir müssen wohl die Ursache solcher Wertungsschwankungen und -Wandlungen ausschließlich in uns selbst suchen. Denn vielleicht haben uns erst die schweren Erschütterungen der letzten Jahre heute empfänglicher undreifergemacht für die Wiedergewinnung mancher der verlorenen Wahrheiten, welche Aufstieg und Blüte des Abendlandes bedingten. Und zu diesen müßte auch die Erkenntnis gehören, daß es letzten Endes nur zwei geistige Belange gibt, aus denen stets seit der Antike wahrhaft große Kunst hervorgehen konnte: das ewig gültige Bild der Gottheit und das ewig veränderliche des Menschen.

Von dem letzteren, dem ,humanistischen“ war die ganze musikalische Entwicklung seit der Klassik einseitig beherrscht. Daß hingegen Bachs 
Werk erst unserer Zeit zugänglich geworden ist, kann nur dahin gedeutet werden, daß erst wir jetzt fähig geworden sind, die sakrale Grundlage, aus der sein gesamtes Schaffen in aller seiner Vielfältigkeit erwachsen ist, zu erkennen und entsprechend einzuschätzen. Denn es handelt sich hier um geistige Werte, die der Musik seit über zweihundert Jahren verlorengegangen waren, deren Beachtung in der gegenwärtigen Situation vielleicht dann einen Ausweg und eine Erfüllung bedeuten kann, wenn es gelingt, dem neuen Kunstwerk wiederum jene umfassende, symbolhafttranszendente Weite zu verleihen, mit der uns Bach die Nähe des Göttlichen gekündet hat. 
